\title{
PIECEWISE CONTINUOUS ALMOST PERIODIC FUNCTIONS AND MEAN MOTIONS
}

\author{
$\mathrm{BY}$ \\ JINGBO XIA
}

\begin{abstract}
In this paper, we prove the existence of mean motion for certain noncontinuous almost periodic functions.
\end{abstract}

Introduction. What we shall investigate in this paper, the existence of mean motion, is an old problem in analysis with a history of two hundred years. In his study of planetary orbits, Lagrange first treated the mean motion for an exponential polynomial of two terms [10]. Later this problem of the existence of mean motion was formulated for analytic almost periodic functions in strips and solved under various assumptions $[\mathbf{8}, \mathbf{9}, \mathbf{1 3}]$. The most remarkable result is due to B. Jessen and H. Tornehave [9]. They introduced what they called the Jensen function $\varphi_{f}(\sigma)=$ $M_{t}\{\log |f(\sigma+i t)|\}$ for an almost periodic function $f$ in a strip $(\alpha, \beta)$ and proved that if $f$ has finite integral base and analytic spatial extension, then the mean motion for $f(\sigma+\cdot)$ exists and equals $\frac{1}{2}\left(\varphi_{f}^{\prime}(\sigma-0)+\varphi_{f}^{\prime}(\sigma+0)\right)$. But for a continuous almost periodic function $f$ which does not have a value arbitrarily close to 0 , it is fairly easy to prove, just by using the almost periodicity, the existence of the mean motion of $f$; see $[\mathbf{9}$, Theorem $\mathbf{1}]$.

In recent studies of singular integral operators with almost periodic symbol, the mean motion of the symbol has been related to analytical invariants in various ways; see [4-6 and 14]. It is this analytical invariant connection of mean motion that motivates the present work. We studied Wiener-Hopf operators with a piecewise continuous, almost periodic symbol in [14]. Among other things, we found that the discontinuities of the symbol do affect the Fredholmness, in the sense of Breuer [3], of the corresponding operator, and that in order to describe the Breuer index of the operator in terms of the average winding number of the symbol, certain curves that join the jumps of the symbol must be taken into account. These findings suggest what the appropriate definition of mean motion for a piecewise continuous, almost periodic function should be. In [14], we gave the definition of mean motion for these functions and proved the existence for invertible elements in certain subalgebras $C\left(S_{\Lambda}\right)$ of symbols. The technique we used there was the construction of a path in the invertibles that joins a given function and a continuous almost periodic function with the same mean motion.

In this paper we take up this matter and prove the existence of mean motion for a broader class of piecewise continuous almost periodic symbols. Since what we have in mind is to link mean motion with analytic index, it is most desired

Received by the editors December 6, 1983 and, in revised form, June 15, 1984.

1980 Mathematics Subject Classification. Primary 43A60; Secondary 46J35, 47 A53.

Key words and phrases. Almost periodic function, mean motion, topological index, analytical invariants. 
that such a proof provide information about path components. Unfortunately, the accumulations of discontinuities prevent us from constructing any kind of path. Our proof of the existence of mean motion depends solely upon the almost periodicity.

1. Preliminaries. We shall first repeat the definition of piecewise continuous almost periodic function we gave in $[\mathbf{1 4}]$. Let $\operatorname{CAP}(\mathbf{R})$ be the $C^{*}$-algebra of continuous almost periodic functions on $\mathbf{R}$. For $\omega>0$ and $b \in \mathbf{R}$, let

$$
\eta_{\omega, b}(t)=\sum_{n \neq 0} \frac{1}{n} \exp [2 n \pi i(t-b) / \omega] .
$$

We denote by $S_{0}$ the algebra of functions $\sum_{j} \prod_{k} f_{j k}$ where $f_{j k}$ is either in $\operatorname{CAP}(\mathbf{R})$ or some $\eta_{\omega, b}$, and by $S$ its uniform closure in $L^{\infty}(\mathbf{R})$. The elements in $S$ can be viewed as generalized almost periodic functions in the following two ways. First, let $\mathbf{R}^{B}$ be the Bohr compactification of $\mathbf{R}$ and let $m$ be the normalized Haar measure on $\mathbf{R}^{B}$, which is induced by the mean value functional on $\operatorname{CAP}(\mathbf{R})$; then each $\eta_{\omega, b}$ is in a natural way identified with the function

$$
\sum \frac{1}{n} \exp (-2 n \pi i b / \omega)[2 n \pi / \omega](\cdot)
$$

in $L^{2}\left(\mathbf{R}^{B}, m\right)$, where each $\lambda \in \mathbf{R}_{d}=\left(\mathbf{R}^{B}\right)^{\prime}$ is identified with the corresponding character on $\mathbf{R}^{B}$. Second, $\eta_{\omega, b}$ is obviously almost periodic in the sense of Riemann and Stepanoff (see [1]). Although it is trivial, we still would like to make it clear that $\eta_{\omega, b}$ is a periodic function with the only jump discontinuities at $\{n \omega+b: n \in \mathbf{Z}\}, \lim _{t \rightarrow n \omega+b-0} \eta_{\omega, b}(t)=\pi i$ and (as definition) $\eta_{\omega, b}(n \omega+b)=$ $\lim _{t \rightarrow n \omega+b+0} \eta_{\omega, b}(t)=-\pi i$. As we explained in $[\mathbf{1 4}], \eta_{\omega, b}$ can be viewed as being a piecewise continuous function only on $\mathbf{R}$, and on $\mathbf{R}^{B}$ its discontinuities have accumulation. Nevertheless, we shall call functions in $S$ piecewise continuous almost periodic functions. Evidently, $S$ contains all piecewise continuous periodic functions of all periods.

LEMMA 1.1. Each $f \in S_{0}$ belongs to a subalgebra generated by $\operatorname{CAP}(\mathbf{R})$ and $\left\{\eta_{\omega_{1}, b_{1}}, \ldots, \eta_{\omega_{k}, b_{k}}\right\}$ where for $i \neq j$ either $\omega_{i}=\omega_{j}$ or $\omega_{i}$ and $\omega_{j}$ are linearly independent over $\mathbf{Q}$. Furthermore, each $f \in S_{0}$ can be expressed as the sum of functions $g \eta_{\lambda_{1}} \cdots \eta_{\lambda_{l}, a_{l}}$ where $\lambda_{1}, \ldots, \lambda_{l}$ are pairwise independent over $\mathbf{Q}$.

The proof is trivial and can be found in $[\mathbf{1 4}, \S 1]$.

A set $E \subset \mathbf{R}$ is said to be relatively dense (r.d. for short) if there exists a number $l>0$ such that $E \cap(t, t+l) \neq \emptyset$ for any $t \in \mathbf{R}$. Let $f$ be a function defined on $\mathbf{R}$ and let $\varepsilon>0$. We shall denote by $E(\varepsilon, f)$ the set of all $\tau$ such that $|f(t+\tau)-f(t)|<\varepsilon$ for all $t \in \mathbf{R}$. $f$ being a continuous almost periodic function is equivalent to that $E(\varepsilon, f)$ is r.d. for all $\varepsilon>0$.

LEMMA 1.2. Let $f_{1}, \ldots, f_{n} \in \operatorname{CAP}(\mathbf{R})$. For any $\varepsilon>0, E\left(\varepsilon, f_{1}\right) \cap \cdots \cap E\left(\varepsilon, f_{n}\right)$ is r.d.

Theorem 11 of $[\mathbf{1}, \S 1]$ provides the proof for the case $n=2$. Unfortunately, we cannot directly quote that result as the proof for Lemma 1.2.

Proof. We use induction. Assume that the result is true for $n \geq 2$. Let $\operatorname{CAP}\left(\mathbf{R}, \mathbf{C}^{n}\right)$ be the uniform closure of polynomials $\left\{\sum a_{j} e^{i \lambda_{j} t}: a_{j} \in \mathbf{C}^{n}, \lambda_{j} \in \mathbf{R}\right\}$ in $L^{\infty}\left(\mathbf{R}, \mathbf{C}^{n}\right)$. For $f \in \operatorname{CAP}\left(\mathbf{R}, \mathbf{C}^{n}\right)$, we can similarly define $E(\varepsilon, f)$ where the 
absolute value is replaced by the norm in $\mathbf{C}^{n}$. The induction hypothesis implies that for every $f \in \operatorname{CAP}\left(\mathbf{R}, \mathbf{C}^{n}\right), E(\varepsilon, f)$ is r.d. To prove the lemma, it suffices to show that $E(\varepsilon, f) \cap E(\varepsilon, g)$ is r.d. for $f, g \in \operatorname{CAP}\left(\mathbf{R}, \mathbf{C}^{n}\right)$. We first prove that for $f, g \in$ $\operatorname{CAP}\left(\mathbf{R}, \mathbf{C}^{n}\right)$ and $\varepsilon, \delta>0, E(\varepsilon, f) \cap\{y: d(y, E(\varepsilon . g)) \leq \delta\}$ is r.d. By the hypothesis, we can find an $l>0, \tau_{n} \in E(\varepsilon / 2, f) \cap((n-1) l, n l)$ and $\sigma_{n} \in E(\varepsilon / 2, g) \cap((n-1) l, n l)$ for every $n \in \mathbf{Z}$. Therefore we have $\tau_{n}-\sigma_{n} \in(-l, l)$. We may assume that $[-l, l)=\bigcup_{j=-k+1}^{k}[(j-1) \delta, j \delta)$. Every $\tau_{n}-\sigma_{n}$ belongs to some $[(j-1) \delta, j \delta)$, therefore there is an $n_{0}>0$ such that for any $n \in \mathbf{Z}$, there is an $n^{\prime}$ between $-n_{0}$ and $n_{0}$ such that $\tau_{n}-\sigma_{n}=\tau_{n^{\prime}}-\sigma_{n^{\prime}}+\theta \delta(|\theta| \leq 1)$ or $\tau_{n}-\tau_{n^{\prime}}=\sigma_{n}-\sigma_{n^{\prime}}+\theta \delta$. But $\tau_{n}-\tau_{n^{\prime}} \in E(\varepsilon, f), \sigma_{n}-\sigma_{n^{\prime}} \in E(\varepsilon, g)$ and the distance between two consecutive $\tau_{n}-\tau_{n^{\prime}}$ is less than $\left(2 n_{0}+3\right) l$. Therefore $E(\varepsilon, f) \cap\{y: d(y, E(\varepsilon, g)) \leq \delta\}$ is r.d. Now let $\varepsilon>\varepsilon_{1}>0$. Then as the scalar case, we can find $\delta>0$ such that $E(\varepsilon, g) \supset$ $\left\{y: d\left(y, E\left(\varepsilon_{1}, g\right)\right) \leq \delta\right\}$. Hence $E(\varepsilon, f) \cap E(\varepsilon, g) \supset E\left(\varepsilon_{1}, f\right) \cap\left\{y: d\left(y, E\left(\varepsilon_{1}, g\right)\right) \leq \delta\right\}$ is r.d. This completes the proof.

Let $\omega>0$ and $b \in \mathbf{R}$. For each $\varepsilon>0$, we denote by $E(\varepsilon ; \omega, b)$ the collection of numbers $l$ such that $d(\mathbf{Z} \omega+b+l, \mathbf{Z} \omega+b)<\varepsilon$.

Corollary 1.3. Let $f_{1}, \ldots, f_{n} \in \operatorname{CAP}(\mathbf{R}), \omega_{i}>0$ and $b_{i} \in \mathbf{R}, i=1, \ldots, m$. Then for any $\varepsilon>0, E\left(\varepsilon ; \omega_{1}, b_{1}\right) \cap \cdots \cap E\left(\varepsilon ; \omega_{m}, b_{m}\right) \cap E\left(f_{1}, \varepsilon\right) \cap \cdots \cap E\left(f_{n}, \varepsilon\right)$ is r.d.

Proof. For fixed $\omega>0$ and $b \in \mathbf{R}$, we define

$$
\xi(t)= \begin{cases}1-2(t-k \omega-b) / \omega: & k \omega+b \leq t<k \omega+b+\frac{1}{2} \omega \\ 2(t-k \omega-b) / \omega-1: & k \omega+b+\frac{1}{2} \omega \leq t<(k+1) \omega+b .\end{cases}
$$

$\xi$ is a continuous periodic function and it is easy to see that

$$
E(\varepsilon ; \omega, b) \supset E(\xi, 2 \varepsilon / \omega) .
$$

From this and Lemma 1.2 the corollary follows immediately.

Lemma 1.4. Let $l \in E(\varepsilon ; \omega, b)$. Then

$$
\left|\eta_{\omega, b}(t+l)-\eta_{\omega, b}(t)\right| \leq \varepsilon / \omega
$$

if $k \omega+b+\varepsilon<t<(k+1) \omega+b-\varepsilon$.

We omit the trivial proof of this lemma.

For a piecewise continuous function $f$ on $\mathbf{R}$, it is generally not possible to define a continuous $\arg f$ on $\mathbf{R}$. But if we also consider the line segments that join $f\left(t_{j}-0\right)$ and $f\left(t_{j}+0\right)$, where $t_{j}$ 's are discontinuities of $f$, there is a natural way to define $\arg f(t)$ on $\mathbf{R}$. Suppose that $f$ does not vanish on $\mathbf{R}$, and none of the abovementioned segments contains 0 . Assume that we have defined $\arg f$ on $\left[t_{i}, t_{j}\right)$. Then we define $\arg f\left(t_{j}\right)=\arg g(1)$ where $g(s)=(1-s) f\left(t_{j}-0\right)+s f\left(t_{j}\right), \arg g(s)$ is continuous on $[0,1]$ and $\arg g(0)=\arg f\left(t_{j}-0\right)$. In this way, we can define $\arg f(t)$ on $\mathbf{R}$ which is in general not continuous.

We can describe this $\arg f$ in a different way. Let $X$ be the topological space of set $\mathbf{R} \times[0,1]$ equipped with the topology generated by open sets $((a, b) \times[0,1]) \cup$ $(\{b\} \times[0, t)),((c, d) \times[0,1]) \cup(\{c\} \times(s, 1])$ and $\{x\} \times(u, v)$. Let

$$
f^{\#}(t, s)=(1-s) f(t-0)+s f(t+0)
$$


then $f^{\#}$ is continuous on $X$. If $f$ is continuous at $t$, then $f^{\#}(t, \cdot)$ is constant on $[0,1]$. It is easy to see that the assumption about $f$ implies that $f^{\#}$ does not vanish. Therefore we are able to define $\arg f^{\#}(t, s)$ as a continuous function on $X \bmod 2 \pi$. If $t_{j}$ is a discontinuity of $f$, then $f^{\#}\left(t_{j}, s\right), 0 \leq s \leq 1$, represents the segment joining $f\left(t_{j}-0\right)$ and $f\left(t_{j}+0\right)$. It is then easy to see that $\arg f^{\#}(t, s)=\arg f(t) \bmod 2 \pi$.

If we generalize this idea somewhat, then for piecewise continuous functions $f_{j k}, j=1, \ldots, n, k=1, \ldots, m$, we define $\arg \sum_{j} \prod_{k} f_{j k}^{\#}(t, s) \bmod 2 \pi$ as a continuous function on $X$ provided $\sum_{j} \prod_{k} f_{j k}^{\#}$ does not vanish on $X$. Let $\left\{f_{n}\right\}$ be a sequence of uniformly bounded piecewise continuous functions on $\mathbf{R}$ and suppose that $\lim _{n \rightarrow \infty}\left\|f_{n}-f\right\|_{\infty}=0$. Obviously we also have $\lim _{m, n \rightarrow \infty}\left\|f_{m}^{\#}-f_{n}^{\#}\right\|_{\infty}=0$ on $X$. Let $f^{\#}$ be the uniform limit of $\left\{f_{n}^{\#}\right\}$, then $f^{\#}$ is bounded and continuous on $X$. This means that $(1-s) f(t-0)+s f(t+0)=f^{\#}(t, s)$ is continuous on $X$. Therefore we generalize the operation \# to uniform limiting functions of piecewise continuous functions.

We denote by $C(S)$ the $C^{*}$-algebra generated by functions $\sum_{j} \prod_{k} f_{j k}^{\#}$ where $f_{j k} \in S$. Let $C_{0}$ be the collection of functions $\sum_{j} \prod_{k} f_{j k}^{\#}$ where $f_{j k} \in S_{0}$; then by the observation above $C_{0}$ is dense in $C(S)$. Denote the invertible elements in $C(S)$ by inv $C(S)$. We define $\arg$ for functions in inv $C(S)$ as follows. Let $\psi \in \operatorname{inv} C(S)$ and let $\left\{\psi_{n}\right\} \subset \operatorname{inv} C(S) \cap C_{0}$ be such that $\left\|\psi-\psi_{n}\right\| \rightarrow 0$ as $n \rightarrow \infty$. Fix a point $\left(t_{0}, s_{0}\right) \in X$, we choose a branch of $\arg \psi_{n}(t, s)$ such that $\mid \arg \psi_{n}\left(t_{0}, s_{0}\right)-$ $\arg \psi_{m}\left(t_{0}, s_{0}\right) \mid<\pi / 2$ for all $m, n$. Routine checking shows that $\arg \psi_{n}$ converges uniformly to a continuous function, which we shall denote by $\arg \psi$, on $X$.

It is also easy to see that $\arg \psi$ does not depend on the choice of $\left\{\psi_{n}\right\}$ and $\psi=|\psi| \exp (i \arg \psi)$. Let $h(\lambda, \mu)$ be a function defined on $\mathbf{R} \times \mathbf{R}$. By the notation $\lim _{\lambda-\mu \rightarrow \infty} h(\lambda, \mu)=c$, we shall mean that for given $\varepsilon>0$ there exists $N>0$ such that $|h(\lambda, \mu)-c|<\varepsilon$ if $|\lambda-\mu| \geq N$. In a similar way we define $\overline{\lim }_{\lambda-\mu \rightarrow \infty} h(\lambda, \mu)$ and $\underline{\lim }_{\lambda-\mu \rightarrow \infty} h(\lambda, \mu)$. For each $\psi \in \operatorname{inv} C(S)$, we define the mean motion of $\psi$ to be

$$
\lim _{\lambda-\mu \rightarrow \infty} \frac{1}{\lambda-\mu}\left(\arg \psi(\lambda, s)-\arg \psi\left(\mu, s^{\prime}\right)\right)
$$

provided the limit exists and does not depend on $s$ and $s^{\prime}$.

The main goal of this paper is to prove the existence of the above limit and its independence on $s$ and $s^{\prime}$. Note that if $\psi=f^{\#}, f \in \operatorname{CAP}(\mathbf{R})$ and $f$ does not vanish on $\mathbf{R}^{B}$, the above limit exists and coincides with the usual mean motion of $f$. This shows that the mean motion we defined for $\psi \in \operatorname{inv} C(S)$ is a natural generalization of the usual mean motion. In [14], we showed the existence of the mean motion for invertible elements in some important subalgebras $C\left(S_{\Lambda}\right)$ of $C(S)$. There we actually presented a path which joins a $\psi \in \operatorname{inv} C\left(S_{\Lambda}\right)$ with an $f^{\#}$ where $f \in \mathrm{CAP}(\mathbf{R})$ has the same mean motion. Thus mean motion serves as a topological index for $C\left(S_{\Lambda}\right)$. If $\mathcal{M}_{\Lambda}$ is the maximal ideal space of $C\left(S_{\Lambda}\right)$, the Cech cohomology $H^{1}\left(\mathcal{M}_{\Lambda}, \mathbf{Z}\right)$ is identified with the real numbers via

$$
H^{1}\left(\mathcal{M}_{\Lambda}, \mathbf{Z}\right)=C\left(S_{\Lambda}\right)^{d} \stackrel{\text { mean motion }}{\longrightarrow} \mathbf{R} .
$$


2. The existence of mean motions. The main result of this paper is the following

THEOREM 1. Every $\psi \in \operatorname{inv} C(S)$ possesses a mean motion

$$
w(\psi)=\lim _{\lambda-\mu \rightarrow \infty} \frac{1}{\lambda-\mu}\left[\arg \psi(\lambda, s)-\arg \psi\left(\mu, s^{\prime}\right)\right],
$$

where the limit is independent of $s$ and $s^{\prime}$. Furthermore, for $\psi, \varphi, \varphi_{0} \in \operatorname{inv} C(S)$, the mean motions satisfy

(a) $w(\psi \varphi)=w(\psi)+w(\varphi)$

(b) $w(\bar{\psi})=-w(\psi)$, and

(c) $w\left(\psi+\varphi_{0}\right)=w(\psi)$

if $\left\|\varphi_{0}\right\|$ is small enough.

This theorem stops short of stating that two elements in inv $C(S)$ with the same mean motion are in the same path component, a property possessed by $\operatorname{CAP}(\mathbf{R})$. Our study is motivated by the connection of mean motion with analytical index of Wiener-Hopf operators $[4,6,14]$. Seeking a relation between mean motion and path components is an important part of the index calculation. In the present situation, we do not have any particular reason to believe that the path components of inv $C(S)$ are determined by the mean motion.

Clearly, the properties (a), (b) and (c) follow from trivial calculation. The only thing that needs a proof is the existence of the limit in the statement of the theorem. We divide the technical steps of the proof into nine lemmas.

Although our main interest is the existence of the mean motion for functions in inv $C(S)$, we shall choose a slightly more general approach to the problem. For each piecewise continuous function $f$ on $\mathbf{R}$, we denote by $D(f)$ the collection of discontinuities of $f$. For each $\varepsilon>0$, we denote by $F(f, \varepsilon)$ the collection of numbers $l$ such that for each $t \in D(f)$ there is $t^{\prime} \in D(f)$ such that $\left|t+l-t^{\prime}\right|<\varepsilon$, and such that $|f(s+l)-f(s)|<\varepsilon$ if $d(D(f), s) \geq \varepsilon$. Let $C=\left\{f_{1}, \ldots, f_{n}\right\}$ be a finite collection of piecewise continuous functions satisfying the following conditions:

(i) Every $f_{j}$ is bounded.

(ii) There exist $N>0$ and $d>0$ such that $\left|f_{j}(t)-f_{j}\left(t^{\prime}\right)\right| \leq N$ if $\left|t-t^{\prime}\right| \leq d, j=$ $1, \ldots, n$.

(iii) For any given $\varepsilon>0$, there exists a $\delta>0$ such that $\left|f_{j}(t)-f_{j}(s)\right|<\delta$ if $|t-s| \leq \delta$ and $[t, s] \cap D\left(f_{j}\right)=\emptyset$.

(iv) For any $j$ and $\varepsilon>0$, if $L \in F\left(f_{j}, \varepsilon\right)$ then $-L \in F\left(f_{j}, \varepsilon\right)$. We denote by \#E the cardinality of set $E$.

(v) For any $i \neq j$, \# $\left(D\left(f_{i}\right) \cap D\left(f_{j}\right)\right)<\infty$.

(vi) $\inf \left\{\left|t-t^{\prime}\right|: t, t^{\prime} \in D\left(f_{j}\right), t \neq t^{\prime}\right\}=\delta_{j}>0, j=1, \ldots, n$. Denote $\delta_{0}=$ $\min \left\{\delta_{1}, \ldots, \delta_{n}\right\}$.

(vii) For every $\varepsilon>0, F\left(f_{1}, \varepsilon\right) \cap \cdots \cap F\left(f_{n}, \varepsilon\right)$ is r.d.

(viii) For every $\sigma>0$, there exists a $\delta>0$ such that

$$
\varlimsup_{\lambda-\mu \rightarrow \infty} \frac{1}{\lambda-\mu} \#\left(E_{\delta} \cap[\mu, \lambda]\right) \leq \sigma
$$

where $E_{\delta}=\bigcup_{j=1}^{n} E_{j}, E_{j}=\left\{t: t \in D\left(f_{j}\right), d\left(t, \bigcup_{i \neq j} D\left(f_{i}\right)\right) \leq \delta\right\}$. 
Now we fix $\psi=\sum_{j} \prod_{k} f_{j k}$ where $\left\{f_{j k}\right\}=\left\{f_{1}, \ldots, f_{n}\right\}$ and we assume that

(ix) $|\psi(t, s)| \geq a>0$ for $t \in R \backslash\left\{t_{1}, \ldots, t_{l}\right\}$ and $s \in[0,1]$. We denote these exceptional points $t_{j}$ by $E_{0}$.

It is possible that $\psi \mid\left\{t_{1}, \ldots, t_{l}\right\} \times[0,1]$ has zeros. Therefore we may not be able to

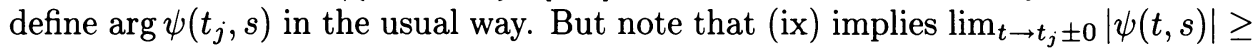
$a>0$. Hence we alter $\psi$ on the vertical segments $\left\{t_{1}, \ldots, t_{l}\right\} \times[0,1]$ such that the new $\psi$ is still continuous on $X$ and $|\psi(t, s)| \geq a>0$ for all $t \in \mathbf{R}$ and $s \in[0,1]$. This enables us to define a version of continuous $\arg \psi$ on $X$. Obviously we have

LEMMA 2.1. The existence and the value of the limit

$$
\lim _{\lambda-\mu \rightarrow \infty} \frac{1}{\lambda-\mu}\left(\arg \psi(\lambda, s)-\arg \psi\left(\mu, s^{\prime}\right)\right)
$$

is independent of the alteration of $\psi$ on $\left\{t_{1}, \ldots, t_{l}\right\} \times[0,1]$.

LEMMA 2.2. There exist $N>0$ and $d>0$ such that for any $s, s \in[0,1]$,

$$
\left|\arg \psi(t, s)-\arg \psi\left(t^{\prime}, s^{\prime}\right)\right| \leq N
$$

if $\left|t-t^{\prime}\right| \leq d$.

LEMMA 2.3. The limit

$$
\varlimsup_{\lambda-\mu \rightarrow \infty} \frac{1}{\lambda-\mu}\left(\arg \psi(\lambda, s)-\arg \psi\left(\mu, s^{\prime}\right)\right)
$$

is finite and independent of $s$ and $s^{\prime}$.

These two lemmas are simple consequences of (i)-(v), (ix) and Lemma 2.1 and we omit the proofs.

LEMMA 2.4. Let $\varepsilon>0$ be given. There is a $\delta>0$ such that if $-\infty<a<$ $b<+\infty,(a, b) \cap\left(\bigcup_{j=1}^{n} D\left(f_{j}\right)\right)=\emptyset,\left|\theta_{1}\right|<\delta,\left|\theta_{2}\right|<\delta, L \in \bigcap_{j=1}^{n} F\left(f_{j}, \delta\right)$ and $\left(a+L+\theta_{1}, b+L+\theta_{2}\right) \cap\left(\bigcup_{j=1}^{n} D\left(f_{j}\right)\right)=\emptyset$, then $|(\arg \psi(x, s)-\arg \psi(x+L, s))+2 k \pi|<$ $\varepsilon$ for $x \in(a, b) \cap\left(a+\theta_{1}, b+\theta_{1}\right)$, where $k$ may depend on the interval $(a, b)$.

Proof. (iii) implies that for any given $\sigma>0$, there is an $\eta>0$ such that if $\left|\theta_{1}\right|<\eta,\left|\theta_{2}\right|<\eta, L \in \bigcap_{j=1}^{n} F\left(f_{j}, \delta\right)$ and $\left(a+L+\theta_{1}, b+L+\theta_{2}\right) \cap\left(\bigcup_{j=1}^{n} D\left(f_{j}\right)\right)=\emptyset$, then $|\psi(x, s)-\psi(x+L, s)|<\sigma$ for $x \in(a, b) \cap\left(a+\theta_{1}, b+\theta_{1}\right)$. By (ix), we have $|(\arg \psi(x, s)-\arg \psi(x+L, s)) \bmod 2 \pi| \leq 2 M \sigma / a$ where $M=\|\psi\|_{\infty}$ on $X$. From this the lemma follows immediately.

LEMMA 2.5. Let $\varepsilon>0$ be given. There is a $\delta>0$ such that if $|a-b|<\delta$ and $(a, b) \cap\left(\bigcup_{j=1}^{n} D\left(f_{j}\right)\right)=\emptyset$, then $|\arg \psi(a, s)-\arg \psi(b, s)|<\varepsilon$.

This is an obvious consequence of (iii) and (ix).

LEMMA 2.6. Let $\varepsilon>0$ and $\delta_{0} / 4>\sigma>0$ be given. There exists a $\delta>0$ such that if $\tilde{t} \in D\left(f_{j}\right) \backslash E_{0}, d\left(\tilde{t}, \bigcup_{i \neq j} D\left(f_{i}\right)\right) \geq \sigma, L \in \bigcap_{j=1}^{n} F\left(f_{j}, \delta\right)$ and $t^{\prime}=$ $\tilde{t}-\sigma / 2, \tilde{t}+\sigma / 2=t^{\prime \prime}$, then

$$
\left|\left[\arg \psi\left(t^{\prime \prime}, s\right)-\arg \psi\left(t^{\prime}, s\right)\right]-\left[\arg \psi\left(t^{\prime \prime}+L, s\right)-\arg \psi\left(t^{\prime}+L, s\right)\right]\right|<\varepsilon .
$$

Proof. Let $\tilde{t} \in D\left(f_{j}\right) \backslash E_{0}$; then near $\tilde{t}$ we have $\psi(t, s)=u_{0}(t)+u_{1}(t) f_{j}^{\#}(t, s)+$ $\cdots+u_{l}(t)\left[f_{j}^{\#}(t, s)\right]^{l}$ where $u_{i}$ 's are continuous functions in a neighborhood of $\tilde{t}$. By 
definition, $f_{j}^{\#}(\tilde{t},[0,1])=\left[f_{j}(\tilde{t}-0), f_{j}(\tilde{t}+0)\right]$ where $[$,$] denotes the line segment$ joining $f_{j}(\tilde{t}-0)$ and $f_{j}(\tilde{t}+0)$. Because of (ix), there is a $\delta_{1}>0$, which is independent of $\tilde{t}$, such that if $\left|u-f_{j}(\tilde{t}-0)\right|<\delta_{1},\left|v-f_{j}(\tilde{t}+0)\right|<\delta_{1}$ and $\left|a_{j} u_{j}(\tilde{t})\right|<\delta_{1}$, then

$$
|[\arg p(1)-\arg p(0)]-[\arg \psi(\tilde{t}, 1)-\arg \psi(\tilde{t}, 0)]|<\varepsilon / 5
$$

where $p(s)=a_{0}+a_{1}[(1-s) u+s v]+\cdots+a_{l}[(1-s) u+s v]^{l}$. If $\delta^{\prime}<\delta_{0} / 4$, then for each $L \in F\left(f_{j}, \delta^{\prime}\right)$ there is a unique $\hat{t} \in D\left(f_{j}\right)$ such that $|\tilde{t}+L-\hat{t}|<\delta^{\prime}$. If $d\left(\tilde{t}, \bigcup_{i \neq j} D\left(f_{i}\right)\right) \geq \sigma$, then there is a $\delta_{0} / 4>\delta_{2}>0$ such that $\left|f_{j}(\tilde{t} \pm 0)-f_{j}(\hat{t} \pm 0)\right|<\delta$ (by (iii)) and $\left|u_{i}(\tilde{t})-u_{i}(\hat{t})\right|<\delta_{1}$ (by the definition of $F(f, \delta)$ ) if $L \in \bigcap_{i=1}^{n} F\left(f_{i}, \delta_{2}\right.$ ) and $|\tilde{t}+L-\hat{t}|<\delta_{0} / 4, \hat{t} \in D\left(f_{i}\right)$. Hence it is clear that

$$
|[\arg \psi(\tilde{t}, 1)-\arg \psi(\tilde{t}, 1)]-[\arg \psi(\hat{t}, 1)-\arg \psi(\hat{t}, 0)]|<\varepsilon / 3 .
$$

By Lemmas 2.4 and 2.5, there is $\sigma / 4>\delta_{3}>0$ such that if $L \in \bigcap_{i=1}^{n} F\left(f_{i}, \delta_{3}\right)$, then

$$
\left|\left[\arg \psi\left(t^{\prime \prime}, s\right)-\arg \psi(\tilde{t}, 1)\right]-\left[\arg \psi\left(t^{\prime \prime}+L, s\right)-\arg \psi(\hat{t}, 1)\right]\right|<\varepsilon / 3
$$

and

$$
\left|\left[\arg \psi\left(t^{\prime}, s\right)-\arg \psi(\tilde{t}, 0)\right]-\left[\arg \psi\left(t^{\prime}+L, s\right)-\arg \psi(\hat{t}, 0)\right]\right|<\varepsilon / 3 .
$$

We stress that none of $\delta_{1}, \delta_{2}$ and $\delta_{3}$ depends on $\tilde{t}$. Therefore $\min \left\{\delta_{1}, \delta_{2}, \delta_{3}\right\}$ is the desired $\delta$.

LEMMA 2.7. Let $\varepsilon>0$ and $\delta_{0} / 4>\sigma>0$ be given. There is a $\delta>0$ such that if $d\left(\left\{t_{1}, t_{2}\right\}, \bigcup_{j=1}^{n} D\left(f_{j}\right)\right) \geq \sigma,\left[t_{1}, t_{2}\right] \cap E_{0}=\emptyset$,

$$
d\left(\left[t_{1}, t_{2}\right] \cap D\left(f_{i}\right),\left[t_{1}, t_{2}\right] \cap D\left(f_{j}\right)\right) \geq \sigma
$$

for all $i \neq j$, and $L \in \bigcap_{j=1}^{n} F\left(f_{j}, \delta\right)$, then

$$
\left|\left[\arg \psi\left(t_{2}, s\right)-\arg \psi\left(t_{1}, s\right)\right]-\left[\arg \psi\left(t_{2}+L, s\right)-\arg \psi\left(t_{1}+L, s\right)\right]\right|<\varepsilon .
$$

Proof. Let $D\left(t_{1}, t_{2}\right)=\left(t_{1}, t_{2}\right) \cap\left(\bigcup_{j=1}^{n} D\left(f_{j}\right)\right)$. Suppose that $\left\{t_{1}, t_{2}\right\}$ is a pair satisfying the restrictions in the statement of the lemma. Because of (iv), there is a $\delta_{1}>0$ such that $D\left(t_{1}+L, t_{2}+L\right) \subset \bigcup\left\{(t-\sigma / 8, t+\sigma / 8): t \in D\left(t_{1}, t_{2}\right)\right\}$ if $L \in$ $\bigcap_{j=1}^{n} F\left(f_{j}, \delta_{1}\right)$. By Lemma 2.4, there is a $\delta_{2}>0$ such that if $D\left(t_{1}, t_{2}\right)=\left\{t^{1}, \ldots, t^{l}\right\}$ and $t_{1}<t^{1}<\cdots<t^{l}<t_{2}$, then $\left|[\arg \psi(x, s)-\arg \psi(x+L, s)]+2 k_{i} \pi\right|<\varepsilon / 2$ for $x \in\left[t^{i}+\sigma / 8, t^{i+1}-\sigma / 8\right]$ and $L \in \bigcap_{j=1}^{n} F\left(f_{j}, \delta_{2}\right)$. By Lemma 2.6, there is a $\delta_{3}>0$ such that if $L \in \bigcap_{j=1}^{n} F\left(f_{j}, \delta_{3}\right)$, then

$$
\begin{aligned}
& \mid\left[\arg \psi\left(t^{i}+\sigma / 8, s\right)-\arg \psi\left(t^{i}-\sigma / 8, s\right)\right] \\
& \quad-\left[\arg \psi\left(t^{i}+L+\sigma / 8, s\right)-\arg \psi\left(t^{i}+L-\sigma / 8, s\right)\right] \mid<\frac{\pi}{4}, \quad i=1, \ldots, l .
\end{aligned}
$$

This means that if we take $\delta=\min \left\{\delta_{1}, \delta_{2}, \delta_{3}\right\}$, then all the $k_{i}$ 's must be the same. Again we stress that $\delta_{1}, \delta_{2}, \delta_{3}$ depend only on the given $\varepsilon$ and $\sigma$. We have

$$
\begin{gathered}
\left|\left[\arg \psi\left(t_{2}, s\right)-\arg \psi\left(t_{1}, s\right)\right]-\left[\arg \psi\left(t_{2}+L, s\right)-\arg \psi\left(t_{1}+L, s\right)\right]\right| \\
\leq\left|\left[\arg \psi\left(t_{2}, s\right)-\arg \psi\left(t_{2}+L, s\right)\right]+2 \pi k_{l+1}\right| \\
\quad+\left|\left[\arg \psi\left(t_{1}, s\right)-\arg \psi\left(t_{1}+L, s\right)\right]+2 k_{0} \pi\right|<\varepsilon
\end{gathered}
$$

since $k_{0}=k_{l+1}$. This completes the proof. 
LEMMA 2.8. Let $\varepsilon>0$ be given. There are $H>0$ and $\delta>0$ such that if $\lambda-\mu \geq H$, and $L \in \bigcap_{j=1}^{n} F\left(f_{j}, \delta\right)$, then

$$
\left|\frac{1}{\lambda-\mu}\left[\arg \psi(\lambda, s)-\arg \psi\left(\mu, s^{\prime}\right)\right]-\frac{1}{\lambda-\mu}\left[\arg \psi(\lambda+L, s)-\arg \psi\left(\mu+L, s^{\prime}\right)\right]\right|<\varepsilon .
$$

PROOF. Since we are considering a large denominator $\lambda-\mu$, the effect of $E_{0}$ can certainly be neglected. Fix $d>0$ and $N>0$ in (ii). Let $\delta_{1}>0$ and $H_{1}>0$ be such that $(N+\pi)\left(\#\left(E_{\delta_{1}} \cap[\mu, \lambda]\right)\right) /(\lambda-\mu)<\varepsilon / 2$ for $\lambda-\mu \geq H_{1}$; see (viii). We apply Lemma 2.7 to the case where $\varepsilon=\pi / 4$ and $\sigma=\min \left\{d, \delta_{0} / 8, \delta_{1}\right\}$ and denote the $\delta$ yielded by the lemma by $\delta_{2}$. Then

$$
(\mu, \lambda) \backslash \bigcup\left\{[t-d, t+d]: t \in E_{\delta_{1}} \cap(\lambda, \mu)\right\}=\bigcup_{j=1}^{m}\left(a_{j}, b_{j}\right)
$$

and it is easy to see that we can choose $m$ such that $m \leq \#\left(E_{\delta_{1}} \cap(\mu, \lambda)\right)+1$. If $t \in D\left(f_{k}\right) \cap\left(\bigcup_{j=1}^{m}\left(a_{j}, b_{j}\right)\right)$, then $d\left(t, \bigcup_{i \neq k} D\left(f_{i}\right)\right) \geq \delta_{1} \geq \sigma$. Therefore if $L \in$ $\bigcap_{j=1}^{n} F\left(f_{j}, \delta_{2}\right)$, then by Lemma 2.8

$$
\mid\left[\arg \psi\left(b_{j}, s\right)-\left[\arg \psi\left(a_{j}, s\right)\right]-\left[\arg \psi\left(b_{j}+L, s\right)-\arg \psi\left(a_{j}+L, s\right)\right] \mid<\pi / 4 .\right.
$$

Thus it is clear that

$$
\begin{gathered}
\left|\left[\arg \psi(\lambda, s)-\arg \psi\left(\mu, s^{\prime}\right)\right]-[\arg \psi(\lambda+\mu, s)-\arg \psi(\mu+L, s)]\right| \\
\leq(N+\pi)\left(\#\left(E_{\delta_{1}} \cap[\mu, \lambda]\right)\right)+4 N
\end{gathered}
$$

if $\delta=\min \left\{\delta_{1}, \delta_{2}\right\}$ and $L \in \bigcap_{j=1}^{n} F\left(f_{j}, \delta\right)$. Let $H_{2}>8 N / \varepsilon$ then $H=\max \left\{H_{1}, H_{2}\right\}$ and $\delta$ are the desired numbers.

LEMMA 2.9. Let $\varepsilon>0$ be given. There is $K>0$ such that if $\lambda-\mu \geq K, \lambda^{\prime}-$ $\mu^{\prime} \geq K$ then

$$
\left|\frac{1}{\lambda-\mu}\left[\arg \psi(\lambda, s)-\arg \psi\left(\mu, s^{\prime}\right)\right]-\frac{1}{\lambda^{\prime}-\mu^{\prime}}\left[\arg \psi\left(\lambda^{\prime}, s\right)-\arg \psi\left(\mu^{\prime}, s^{\prime}\right)\right]\right|<\varepsilon .
$$

PROOF. Applying Lemma 2.8 to the number $\varepsilon / 8$, we obtain the corresponding $H>0$ and $\delta>0$ as described in that statement. We fix this $\delta$ and the numbers $d, N$ in (ii). By assumption (vii), there is an $l>0$ such that $\bigcap_{j=1}^{n} F\left(f_{j}, \delta\right)$ has nonempty intersection with every interval whose length is not less than $l$. By (ii), there is an $N_{1}>0$ such that $\left|\arg \psi(x, s)-\arg \psi\left(y, s^{\prime}\right)\right| \leq N_{1}|x-y|$. Let $H_{1}=$ $\max \left\{H, l, 8 l N_{1} / \varepsilon\right\}$ and let $K=8(N+d)\left(H_{1}+2 l+d\right) / \varepsilon d$. We shall prove that this $K$ will do. We fix an $x_{0} \in \mathbf{R}$. Let $\lambda, \mu$ be any numbers such that $\lambda-\mu \geq K$. Suppose we have found $L_{1}, \ldots, L_{k} \in \bigcap_{j=1}^{n} F\left(f_{j}, \delta\right)$ such that $L_{1} \in\left(\mu-x_{0}, \mu-x_{0}+l\right), L_{2} \in$ $\left(L_{1}+H_{1}-x_{0}, L_{1}+H_{1}+l-x_{0}\right), \ldots, L_{k} \in\left(L_{k-1}+H_{1}-x_{0}, L_{k-1}+H-x_{0}+l\right)$. If $\lambda-x_{0}-\left(L_{k}+H_{1}\right) \geq H_{1}+l$, then there is an $L_{k+1} \in \bigcap_{j=1}^{n} F\left(f_{j}, \delta\right) \cap\left(L_{k}+\right.$ $\left.H_{1}-x_{0}, L_{k}+H_{1}-x_{0}+l\right)$. By induction, we obtain $L_{1}, \ldots, L_{m} \in \bigcap_{j=1}^{n} F\left(f_{j}, \delta\right)$ such that $(\lambda-\mu) /\left(H_{1}+l\right) \leq m \leq(\lambda-\mu) / H_{1}, H_{1}<L_{k+1}-L_{k}<H_{1}+2 l$, 


$$
\begin{aligned}
0<L_{1}-\left(\mu-x_{0}\right)<l \text { and } 0 & \leq\left(\lambda-x_{0}\right)-\left(L_{m}+H_{1}\right)<H_{1}+l . \text { Therefore } \\
\arg \psi\left(\mu, s^{\prime}\right)-\arg (\lambda, s)= & \sum_{j=1}^{m}\left(\arg \psi\left(L_{j}+x_{0}, 0\right)-\arg \psi\left(L_{j}+x_{0}+H_{1}, 1\right)\right) \\
& +\sum_{j=1}^{m-1}\left(\arg \psi\left(L_{j}+x_{0}+H_{1}, 1\right)-\arg \psi\left(L_{j+1}+x_{0}, 0\right)\right) \\
& +\left(\arg \psi\left(\mu, s^{\prime}\right)-\arg \psi\left(L_{1}+x_{0}, 0\right)\right) \\
& +\left(\arg \psi\left(L_{m}+x_{0}+H_{1}, 1\right)-\arg \psi(\lambda, s)\right) .
\end{aligned}
$$

We use (j) to denote the $j$ th term of the right-hand side of the above equality. By our setting,

$$
\begin{aligned}
\frac{\mid(1)-}{-} & m\left[\arg \psi\left(x_{0}, 0\right)-\arg \psi\left(x_{0}+H_{1}, 1\right)\right] \mid \\
\lambda-\mu & \sum_{j=1}^{m}\left|\left[\arg \psi\left(L_{j}+x_{0}, 0\right)-\arg \psi\left(L_{j}+H_{1}+x_{0}, 1\right)\right]\right| \\
& -\frac{\left[\arg \psi\left(x_{0}, 0\right)-\arg \psi\left(x_{0}+H_{1}, 1\right)\right]}{\lambda-\mu} \\
& <\frac{\varepsilon m H_{1}}{8(\lambda-\mu)} \leq \frac{\varepsilon}{8} .
\end{aligned}
$$

Since $0<L_{k+1}-\left(L_{k}+H_{1}\right) \leq l$, we have $(|(2)|+|(3)|) /(\lambda-\mu) \leq N_{1} l m /(\lambda-\mu) \leq$ $N_{1} l / H_{1}<\varepsilon / 8$. It is easy to see that $|(4)| /(\lambda-\mu) \leq(N+d)\left(H_{1}+l\right) / d K<\varepsilon / 8$. Therefore if we have another pair $\lambda^{\prime}-\mu^{\prime} \geq K$, then there is $\left(\lambda^{\prime}-\mu^{\prime}\right) /\left(H_{1}+l\right) \leq$ $m^{\prime} \leq\left(\lambda^{\prime}-\mu^{\prime}\right) / H_{1}$ such that

$$
\begin{aligned}
& \left|\frac{1}{\lambda-\mu}\left[\arg \psi(\lambda, s)-\arg \psi\left(\mu, s^{\prime}\right)\right]-\frac{1}{\lambda^{\prime}-\mu^{\prime}}\left[\arg \psi\left(\lambda^{\prime}, \tilde{s}\right)-\arg \psi\left(\mu^{\prime}, \tilde{s}\right)\right]\right| \\
& \quad<\frac{3 \varepsilon}{4}+\left|\frac{m}{\lambda-\mu}-\frac{m^{\prime}}{\lambda^{\prime}-\mu^{\prime}}\right| \mid\left[\arg \psi\left(x_{0}, 0\right)-\arg \psi\left(x_{0}+H_{1}, 1\right) \mid\right. \\
& \leq\left|\frac{m}{\lambda-\mu}-\frac{m^{\prime}}{\lambda^{\prime}-\mu^{\prime}}\right| N_{1} H_{1} .
\end{aligned}
$$

But $m /(\lambda-\mu), m^{\prime} /\left(\lambda^{\prime}-\mu^{\prime}\right) \in\left[1 /\left(H_{1}+l\right), 1 / H_{1}\right]$, so $\left|m /(\lambda-\mu)-m^{\prime} /\left(\lambda^{\prime}-\mu^{\prime}\right)\right| N_{1} H_{1} \leq$ $l N_{1} /\left(H_{1}+l\right)<\varepsilon / 8$. This completes the proof.

If we intepret the above lemma in terms of the limit language, we have

THEOREM 2. If $\psi$ satisfies assumptions (i)-(ix), then the limit

$$
\lim _{\lambda-\mu \rightarrow \infty} \frac{1}{\lambda-\mu}\left[\arg \psi(\lambda, s)-\arg \psi\left(\mu, s^{\prime}\right)\right]
$$

exists and is independent of $s$ and $s^{\prime}$.

ProOF OF THEOREM 1. Clearly, we only need to prove the theorem for $\psi \in \operatorname{inv} C(S) \cap C_{0}$. So we may assume that $\psi=\sum_{j} \prod_{k} f_{j k}^{\#}$ with $f_{j k} \in S_{0}$ and each $f_{j k}$ has the form $\sum_{m} \prod_{n} g_{m n}$ where $\left\{g_{m n}\right\} \subset\left\{\eta_{\omega_{1}, b_{1}}, \ldots, \eta_{\omega_{l}, b_{l}}\right\} \cup \operatorname{CAP}(\mathbf{R})$ 
and $\omega_{1}, \ldots, \omega_{l}$ are described in Lemma 1.1. Note that in general $\prod_{n} g_{m n}^{\#} \neq$ $\left(\prod_{n} g_{m n}\right)^{\#}$. But Lemma 1.1 tells us that $\left(\prod_{n} g_{m n}\right)^{\#}$ defers from $\prod_{n} g_{m n}^{\#}$ only on a set $\left\{t_{1}, \ldots, t_{n}\right\} \times[0,1]$. After certain rearrangements we may assume that on $X \backslash\left\{t_{1}, \ldots, t_{n}\right\} \times[0,1], \psi=\sum_{j} \prod_{k} f_{j k}^{\#}$ where either $f_{j k} \in \operatorname{CAP}(\mathbf{R})$ or $f_{j k}=\eta_{\omega, b}$. Since the values of $\psi$ on $\left\{t_{1}, \ldots, t_{n}\right\} \times[0,1]$ do not affect the existence of mean motion, we simply assume $\psi=\sum_{j} \prod_{k} f_{j k}^{\#}$ on $X$. Let $\left\{f_{j k}\right\}=\left\{g_{1}, \ldots, g_{l} ; \eta_{\lambda_{1}, a_{1}}, \ldots\right.$, $\left.\eta_{\lambda_{m}, a_{m}}\right\}$ where $g_{1}, \ldots, g_{l} \in \operatorname{CAP}(\mathbf{R})$ and for $i \neq j$, either $\lambda_{i}$ and $\lambda_{j}$ are linearly independent or else $d\left(\mathbf{Z} \lambda_{i}+a_{i}, \mathbf{Z} \lambda_{j}+b_{j}\right)>0$. Since originally we chose $\psi \in \operatorname{inv} C(S) \cap C_{0}$, it is easy to verify that the family $\left\{f_{j k}\right\}$ satisfies (i)-(vi) and (ix). (vii) is guaranteed by Corollary 1.3. But the fact that $\left\{f_{j k}\right\}$ also satisfies (viii) is not so obvious. Indeed, to show that (viii) holds for $\left\{f_{j k}\right\}$, it suffices to consider a pair $\left\{\eta_{\omega, b}, \eta_{\lambda, a}\right\}$ where $\omega$ and $\lambda$ are linearly independent over $\mathbf{Q}$. Without loss of generality, we may further assume that $\lambda=1, \omega$ is irrational and $a=0$. Therefore to verify (viii) for $\left\{f_{j k}\right\}$ we only need to show that for given $\sigma>0$, there is a $\delta>0$ such that

$$
\varlimsup_{m-n \rightarrow \infty} \frac{1}{m-n+1} \#\{k: k \omega+b \in[n, m], d(k \omega+b, \mathbf{Z})<\delta\} \leq \sigma .
$$

We shall use ergodic theory to prove this inequality. We identify the unit circle $T^{1}$ with $\mathbf{R} / \mathbf{Z}$ via $t \mapsto t \bmod \mathbf{Z}$. Let $T$ be the irrational ration $T(t \bmod \mathbf{Z})=$ $(t+\omega \bmod \mathbf{Z})$ on $T^{1}$. It is well known that the arc length measure $m$ on $T^{\mathbf{1}}$ is the only $T$-invariant probability Borel measure on $T$. In other words, the action of $T$ on $T^{1}$ is uniquely ergodic. Therefore if $f$ is a Riemann integrable function defined on $T^{1}$

$$
\frac{1}{k} \sum_{j=0}^{k-1} f \circ T^{j}(z) \rightarrow \int_{T^{1}} f(\tau) d m(\tau)
$$

uniformly on $T^{1}$; see [12, p. 156]. Let $E_{\delta}=\{t \bmod \mathbf{Z}:|t \bmod \mathbf{Z}|<\delta\}$ and let $\chi_{E_{\delta}}$ be the characteristic function of $\chi_{E_{\delta}}$. Since $E_{\delta}$ is an open arc, $\chi_{E_{\delta}}$ is Riemann integrable on $T^{1}$. Thus

$$
\begin{aligned}
& \frac{1}{m-n+1} \#\{k: k \omega+b \in[n, m], d(k \omega+b, \mathbf{Z})<\delta\} \\
& \quad=\frac{1}{m-n+1} \sum_{j=n}^{m} \chi_{E_{\delta}}((j \omega+b) \bmod \mathbf{Z}) \\
& \quad=\frac{1}{m-n+1} \sum_{j=0}^{m-n} \chi_{E_{\delta}} \circ T^{j}((n \omega+b) \bmod \mathbf{Z}) \rightarrow m\left(E_{\delta}\right) \\
& =2 \delta
\end{aligned}
$$

uniformly as $m-n \rightarrow \infty$. This shows that $\sigma=2 \delta$ will do. Hence all (i)-(ix) have been verified for $\left\{f_{j k}\right\}$. By Theorem 2, the desired limit exists. This completes the proof.

\section{REFERENCES}

1. A. S. Besicovitch, Almost periodic functions, Cambridge Univ. Press, London and New York, 1932.

2. H. Bohr, Almost periodic functions, Chelsea, New York, 1947. 
3. M. Breuer, Fredholm theories in von Neumann algebras. I, II, Math. Ann. 178 (1968), 243-254; 180 (1969), 313-325.

4. R. Carey and J. Pincus, Mean motion, principal functions and zeros of Dirichlet series, Integral Equations and Operator Theory 2 (1979), 484-502.

5. (1977), 153-218.

6. L. Coburn, R. Douglas, D. Schaeffer and I. Singer, On the $C^{*}$-algebras of operators on a half-space. II. Index theory, Inst. Hautes Études Sci. Publ. Math. 40 (1971), 69-79.

7. R. Doss, On mean motion, Amer. J. Math. 79 (1957), 389-396.

8. P. H. Hartman, Mean motions and almost periodic functions, Trans. Amer. Math. Soc. 46 (1939), 64-81.

9. B. Jessen and H. Tornehave, Mean motions and zeros of almost periodic functions, Acta Math. 77 (1945), 137-279.

10. J. Lagrange, Théorie des variations séculaires des éléments des planètes. I, II, Nouveaux Mémoires de l'Académie de Berlin 1781, 1782, Oeuvres 5, pp. 123-344.

11. D. Schaeffer, An index theorem for systems of difference equations on a half space, Inst. Hautes Études Sci. Publ. Math. 42 (1973), 397-403.

12. K. Petersen, Ergodic theory, Cambridge Univ. Press, Cambridge, 1983.

13. H. Weyl, Mean motion. I, Amer. J. Math. 60 (1938), 889-896; II, Amer. J. Math. 61 (1939), 143-148.

14. J. Xia, Wiener-Hopf operators with piecewrie continuous almost periodic symbol, J. Operator Theory (to appear).

Department of MAThematics, The UNiversity of IOWA, IOWA City, IOWA 52242 